\title{
CONSTITUINTES QUÍMICOS DOS GALHOS DE Simaba guianensis subesp. ecaudata (Cronquist) ${ }^{\#}$
}

Rita de Cássia Saraiva Nunomura*

Departamento de Química, Instituto de Ciências Exatas, Universidade Federal do Amazonas, Av. Gal. Rodrigo Otávio Jordão Ramos, 3000, 69077-000 Manaus - AM, Brasil

Angelo C. Pinto

Instituto de Química, Universidade Federal do Rio de Janeiro, Centro de Tecnologia, Bl. A, Cidade Universitária, 21945-970 Rio de Janeiro - RJ, Brasil

Sergio Massayoshi Nunomura e Adrian Martin Pohlit

Coordenação de Tecnologia e Inovação, Instituto Nacional de Pesquisas da Amazônia, Av. André Araújo, 2936, 69083-000 Manaus - AM, Brasil

Ana Cláudia Fernandes Amaral

Fundação Oswaldo Cruz (FIOCRUZ/Farmanguinhos), Rua Sizenando Nabuco, 100, 21041-250 Rio de Janeiro - RJ, Brasil

Recebido em 26/5/12; aceito em 27/9/12; publicado na web em 26/10/12

\begin{abstract}
CHEMICAL CONSTITUENTS FROM STEMS OF Simaba guianensis subesp. ecaudata (Cronquist). Simaba guianensis subesp. ecaudata (Simaroubaceae) is a tree found in the Brazilian Amazon. This work describes for the first time the fractionation of stems of this species that resulted in the isolation of the cytotoxic triterpene piscidinol A, the alkaloid 9-methoxycanthin-6-one, caryophyllene oxide, also isolated for the first time from this species and a new alkaloid (6-methoxy-(9H- $\beta$-carbolin-1-il)-(Z)-2-propenoic acid). Quantification of 9-methoxycanthin-6-one in different extracts and fractions of stems of S. guianensis by high performance liquid chromatography was also performed. The concentration of 9-methoxycanthin-6-one in methanolic and aqueous extracts were inferior to the known cytotoxic concentration of this compound.
\end{abstract}

Keywords: piscidinol A; 9-methoxycanthin-6-one; HPLC.

\section{INTRODUÇÃO}

A família Simaroubaceae é caracterizada por árvores e arbustos distribuídos em regiões tropicais e subtropicais. Várias espécies dessa família são conhecidas por apresentarem princípios amargos com notáveis propriedades medicinais e são utilizadas popularmente como vermífugas, antivirais e no combate a febres, entre outras propriedades.

O gênero Simaba é o segundo maior gênero da família, com aproximadamente 35 espécies que ocorrem na América do Sul. As espécies desse gênero são encontradas em ambientes bastante diversificados como mata úmida, alagável ou de terra firme, e apresentam interesse por suas propriedades medicinais, devido às substâncias fortemente amargas nelas encontradas, conhecidas como quassinoides..$^{2-4}$

Apesar do grande número de espécies desse gênero, poucas espécies de Simaba foram estudadas quimicamente. Dentre as já estudadas, podem-se destacar as espécies $S$. cedron, S. orinocensis, S. cuspidata, S. cuneata, S. multiflora, S. guianensis e S. polyphylla. De espécies do gênero Simaba foram isolados alcaloides piridoindóis do tipo cantinona, cumarinas, antraquinonas, flavonoides, esteroides, triterpenos e quassinoides. Estes últimos são resultantes da degradação de triterpenos. ${ }^{5}$ A presença de quassinoides e alcaloides do tipo cantinona em espécies desse gênero são de particular importância como marcadores químicos do gênero e da família Simaroubaceae. ${ }^{6}$

As propriedades medicinais das espécies do gênero Simaba foram confirmadas experimentalmente para algumas atividades, entre elas citotóxica, antitumoral (S. cedron, S. multiflora, S. cuspidata), antimalárica (S. cedron, S. guianensis), antiviral (S. africana ou Quassia africana), antimicrobiana (S. undulata) e antiúlcera (S. ferruginea) ${ }^{5}$

*e-mail: ritasn@ufam.edu.br

\#Artigo em homenagem ao Prof. Otto R. Gottlieb (31/8/1920-19/6/2011)
A espécie Simaba guianensis subesp. ecaudata (Cronquist) é caracterizada como um arbusto ou uma pequena árvore de até $10 \mathrm{~m}$ de altura, nativa da região amazônica. ${ }^{7}$ Essa espécie caracteriza-se com folíolos cartáceos, de cor verde-amarelada, brilhantes, com margem levemente ondulada. As suas flores possuem odor agradável, ráquis de inflorescência verde-claro aveludada; pedicelos, pétalas, estames e anteras verde-amareladas. Esses arbustos costumam ser encontrados em matas de terra firme, de solo argiloso.

Visando contribuir para o conhecimento da composição química de espécies do gênero Simaba que ocorrem na Amazônia, este trabalho descreve o isolamento do sesquiterpeno óxido de cariofileno (1), o triterpeno piscidinol A (2), o alcaloide 9-metóxi-cantin-6-ona (3) também isolado de $S$. polyphylla ${ }^{8}$ e, pela primeira vez, o alcaloide 6-metóxi-(9H- $\beta$-carbolin-1-il)-(Z)-prop-2-enoico (4) de Simaba guinanensis subesp. ecaudata (Figura 1). A dosagem de 9-metóxi-cantin-6-ona também foi realizada, considerando-se sua significância biológica e quimiotaxonômica. $^{6}$

\section{PARTE EXPERIMENTAL}

\section{Equipamentos}

Para obtenção de pontos de fusão foi utilizado o aparelho MelTemp II (Lab. Devices Inc., EUA). Os valores não foram corrigidos.

Os espectros de infravermelho foram obtidos em aparelho Nicolet Magna IR 760, com pastilhas comprimidas em $\mathrm{KBr}$ anidro. Os espectros foram calibrados com filmes de poliestireno de $0,05 \mathrm{~mm}$ de espessura.

Os espectros de RMN foram obtidos em aparelho Bruker, modelo DRX-200, operando a $200 \mathrm{MHz}$ para $\mathrm{RMN}{ }^{1} \mathrm{H}$ e a $50 \mathrm{MHz}$ para RMN ${ }^{13} \mathrm{C}$, no aparelho DRX-300, operando a $300 \mathrm{MHz}$ para $\mathrm{RMN}{ }^{1} \mathrm{H}$ e a $75 \mathrm{MHz}$ para $\mathrm{RMN}{ }^{13} \mathrm{C}$ e no aparelho de modelo DRX-500, operando 


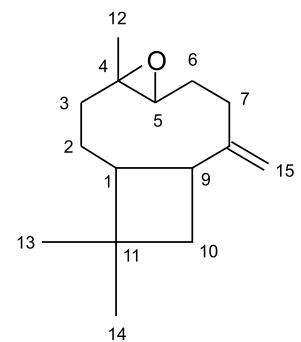

1

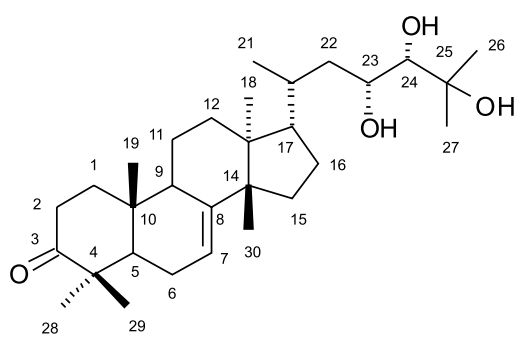

2<smiles>COc1ccc2cccnc2c1-n1c(=O)ccc2ncccc21</smiles>

3

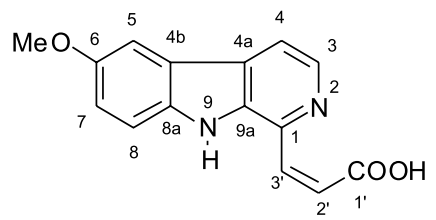

4
Figura 1. Substâncias isoladas de S. guianensis subesp. ecaudata

a $500 \mathrm{MHz}$ para $\mathrm{RMN}{ }^{1} \mathrm{H}$ e $125 \mathrm{MHz}$ para $\mathrm{RMN}{ }^{13} \mathrm{C}$.

Para a obtenção dos espectros de massas de alta resolução foi utilizado o cromatógrafo gás-líquido Hewlett-Packard 6890 Series GC System acoplado ao espectrômetro de massas do tipo Hewlett-Packard HP 5973 Mass Selective Detector. Foi utilizada a coluna DB-5, com $25 \mathrm{~m}$ x 0,2 mm x 0,11 $\mu \mathrm{m}$ de espessura de filme. Temperatura do injetor: $270{ }^{\circ} \mathrm{C}$ com divisor de fluxo (1/20); gás de arraste: He. Temperatura inicial do forno $80^{\circ} \mathrm{C}$, rampa de aquecimento a $8^{\circ} \mathrm{C} / \mathrm{min}$ até $290^{\circ} \mathrm{C}$ permanecendo nessa temperatura por $5 \mathrm{~min}$. $\mathrm{O}$ espectro de massas foi obtido por impacto de elétrons a $70 \mathrm{eV}$. Também foi utilizado, para a análise da substância 4, o espectrômetro de massas TSQ-Thermo, com analisador do tipo triplo-quadrupólo e fonte de ionização tipo APCI.

$\mathrm{O}$ fracionamento por cromatografia líquida de média pressão foi realizado em aparelho de MPLC Chromatography Pump Büchi 688 Gradient Former Büchi 687 com detector de UV/VIS FilterPhotometer Büchi e coletor de frações Büchi 684. Utilizou-se coluna em fase reversa de RP-18 Lichroprep RP-18 (Merck) com partículas de 40-63 $\mu \mathrm{m}$.

A dosagem de 9-metóxi-cantin-6-ona por CLAE foi realizada em aparelho Shimadzu, modelo LC-10, equipado com bomba quaternária, detector de ultravioleta SPD-10AVP, válvula de injeção Rheodyne e Chemstation (CLASS-VP, versão 6.1) e coluna LiChrospher 60

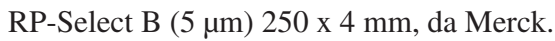

\section{Coleta do material botânico}

As partes aéreas de dois espécimes de Simaba guianensis subesp. ecaudata (Cronquist) foram coletadas no dia 16/2/2002 (período de chuvas), na Reserva Florestal Adolpho Ducke do INPA, localizada na rodovia AM-010 a $23 \mathrm{~km}$ de Manaus. As exsicatas dos espécimes encontram-se no herbário do INPA, identificadas com os números 3861 e 3859. O material vegetal foi seco à sombra e depois separado em galhos e folhas para posterior moagem em moinho de facas.

\section{Preparação dos extratos}

Foram extraídos, com hexano a frio, 2,0 kg de galhos moídos por um período de 7 dias. O procedimento foi repetido por mais duas vezes com a mesma serragem. Os extratos foram reunidos e concentrados em rotaevaporador, resultando em $8,9 \mathrm{~g}$ de extrato. Após extração em hexano, o resíduo foi extraído a frio com $\mathrm{MeOH}$ também por 7 dias.
O procedimento foi repetido por mais duas vezes. A massa resultante do extrato metanólico após concentração em rotaevaporador foi de $63,1 \mathrm{~g}$. Foram separados 58,0 g desse extrato. Esse foi submetido a um fracionamento por partição líquido-líquido com $\mathrm{CHCl}_{3}$ e AcOEt.

\section{Preparação dos extratos aquosos}

Foram obtidos por infusão, adicionando-se ao material vegetal $400 \mathrm{~mL}$ de água fervente e aguardando-se por $15 \mathrm{~min}$. Em seguida, os extratos foram filtrados e concentrados em rotaevaporador e liofilizados.

\section{Isolamento dos constituintes químicos}

O extrato hexânico dos galhos de Simaba guianensis $(8,9 \mathrm{~g})$ foi submetido a uma separação em coluna de gel de sílica com sistema gradiente de hexano/AcOEt. Foram obtidas 69 frações, que foram reunidas em 13 frações após análise em CCD. A fração 1-12 foi submetida a uma separação em coluna de sílica flash com gradiente hexano/AcOEt (0 a 100\% de AcOEt) obtendo-se 22 frações que, após análise por CCD, foram reunidas em 7 frações. A fração 4 (103,0 mg) eluída em hexano foi identificada como sendo o óxido de cariofileno (1), após análises espectroscópicas.

A fração 64-69 do extrato hexânico dos galhos também foi submetida a uma separação em coluna de sílica gel, utilizando $\mathrm{CHCl}_{3} /$ $\mathrm{MeOH}$ como sistema gradiente de eluição, até chegar à concentração de $10 \%$ de $\mathrm{MeOH}$, que após análise por CCD resultou em 10 frações. Em seguida, a fração 3 obtida desse fracionamento foi recristalizada em AcOEt. Os cristais incolores (164,7 mg) após análise de espectros de RMN mono e bidimensionais, EM e IV foram identificados como o triterpeno piscidinol A (2).

A fração clorofórmica resultante do fracionamento do extrato metanólico dos galhos de Simaba guianensis subesp. ecaudata (20,6 g) foi inicialmente submetida a uma separação simples em coluna filtrante de gel de sílica. O eluente inicial foi hexano 100\%, seguido de clorofórmio 100\%, $\mathrm{CHCl}_{3} / \mathrm{MeOH}$ (97:3), $\mathrm{CHCl}_{3} / \mathrm{MeOH}$ (95:5), $\mathrm{CHCl}_{3} / \mathrm{MeOH}$ (7:3) e MeOH $100 \%$, respectivamente. Foram recolhidas 15 frações e a fração 7 indicou a presença de alcaloides, por análise em CCD quando revelado com reagente de Dragendorff. Essa fração foi dissolvida em uma mistura de $\mathrm{MeOH} / \mathrm{H}_{2} \mathrm{O}$ (9:1) e particionada com hexano. A fração hidroalcoólica, $3,15 \mathrm{~g}$, foi fracionada por MPLC em coluna de fase reversa (RP-18) com sistema de eluição em EtOH/ $\mathrm{H}_{2} \mathrm{O}$ de 0 a $70 \%$ de EtOH. Após análise em CCD, as frações iguais foram reunidas em 39 frações. A fração 19 foi recristalizada em $\mathrm{MeOH}$ resultando no isolamento de $62,8 \mathrm{mg}$ do alcaloide 9-metoxi-cantin-6-ona (3), solúvel em $\mathrm{CHCl}_{3}$, e $16,9 \mathrm{mg}$ de outro alcaloide com ponto de fusão $232^{\circ} \mathrm{C}$ identificado como 6-metóxi- $\beta$-carbolin(Z)-propenoico (4), solúvel em MeOH. A fração 30 foi recristalizada em $\mathrm{MeOH}$ a quente e resultou na formação de um material cristalino incolor (29,0 mg) identificado como o triterpeno piscidinol A (2).

\section{Determinação do teor de 9-metóxi-cantin-6-ona por CLAE-DAD}

Os extratos metanólicos, aquosos e as frações clorofórmicas dos galhos de $S$. guianensis foram submetidos a uma análise por cromatografia líquida de alta eficiência (CLAE) para avaliação da concentração de 9-metóxi-cantin-6-ona, presente nesses extratos. As condições de análise foram modo isocrático ACN/solução aquosa de TFA 0,05\% (30:70) por $35 \mathrm{~min}$, fluxo de 1,0 $\mathrm{mL} / \mathrm{min}$ em coluna LiChrospher 60 RP-Select B $(5 \mu \mathrm{m}) 250$ x 4 mm, da Merck. Todas as injeções foram realizadas com volume de loop de $20 \mu \mathrm{L}$. A detecção foi feita por ultravioleta no comprimento de onda de $254 \mathrm{~nm}$. 


\section{Curva de calibração}

A quantificação de 9-metóxi-cantin-6-ona foi realizada usando padrão externo, a partir de uma curva de calibração obtida pela injeção de 5 diferentes concentrações $(0,0625 ; 0,125 ; 0,250 ; 0,500$ e $1,000 \mu \mathrm{g} / \mathrm{mL}$ ) do padrão, em CLAE, nas mesmas condições de análise utilizadas para os extratos e frações dos espécimes estudados. $\mathrm{O}$ padrão utilizado foi isolado conforme descrito anteriormente $\mathrm{e}$ apresentou grau de pureza de 98,55\% determinado por CLAE a 254 nm (método de normalização de área).

\section{Preparação das amostras para análise em CLAE}

Os extratos metanólicos e aquosos foram submetidos a um pré-tratamento por extração em fase sólida antes da análise. As frações clorofórmicas dos galhos de cada um dos espécimes foram apenas solubilizadas em metanol, na concentração de $0,125 \mathrm{mg} / \mathrm{mL}$.

\section{Tratamento dos extratos metanólicos}

Os extratos metanólicos dos galhos $S$. guianensis analisados foram inicialmente solubilizados em uma mistura de $\mathrm{MeOH} / \mathrm{H}_{2} \mathrm{O}$ (1:1) na concentração de $1,0 \mathrm{mg} / \mathrm{mL}$ e volume total de 2,0 mL. Em seguida, foi eluída num cartucho de extração em fase sólida (EFS) de fase reversa (Seppak da Waters Inc.) com 10,0 mL de solução MeOH/ $\mathrm{H}_{2} \mathrm{O}(1: 1)$ gerando a fração 1 , contendo as substâncias mais polares. Em seguida, eluiu-se a amostra com 2,0 mL da solução MeOH/AcOEt (7:3) gerando a fração 2, contendo o analito. Ao final, foi feita uma lavagem de 10,0 mL da mesma solução, gerando a fração 3 .

Os extratos aquosos dos galhos dos espécimes em estudo foram preparados por infusão. A partir dos extratos secos, preparou-se uma solução de $5,0 \mathrm{mg} / \mathrm{mL}$ no volume total de $10,0 \mathrm{~mL}$, que foi submetida a um tratamento por EFS com o mesmo tipo de cartucho empregado anteriormente. Após a eluição da solução do extrato aquoso, que gerou a fração 1, a amostra foi eluída com $10,0 \mathrm{~mL}$ da mistura $\mathrm{MeOH} / \mathrm{H}_{2} \mathrm{O}$ (1:1), gerando a fração 2. A fração 3, contendo o analito, foi gerada pela eluição de 1,0 mL da mistura $\mathrm{MeOH} / \mathrm{AcOEt}$ (7:3) e, em seguida, foi realizada a lavagem do cartucho com $10 \mathrm{~mL}$ da mesma mistura para obtenção da fração 4 .

\section{Caracterização do óxido de cariofileno $(\mathbf{1})^{9}$}

Óleo incolor, IV $(\mathrm{KBr}) v_{\max }\left(\mathrm{cm}^{-1}\right): 3.066,2.950,2.932,2.861$, 1.629. EM-IE $(\mathrm{m} / \mathrm{z})$ : de $220\left(\mathrm{M}^{+}\right), 205$ (4), 177 (10), 149 (14), 121 (33), 93 (79), 79 (95), 69 (61) e 41 (100). RMN ${ }^{1} \mathrm{H}\left(300 \mathrm{MHz}, \mathrm{CDCl}_{3}\right.$ ): $\delta 1,75(\mathrm{t} ; 9,6 \mathrm{~Hz} ; 9,4 \mathrm{~Hz}, \mathrm{H}-1) ; 1,29(\mathrm{sl}, \mathrm{H}-2) ; 2,10$ (m , H-3), 1,08 (d; $5,7 \mathrm{~Hz}, \mathrm{H}-3$ ), 2,86 (dd; 3,9 Hz; 10,5 Hz, H-5); 2,10(m, H-6); 2,29 (m, H-6); 2,10 (m, H-7); 2,30 (m, H-7); 2,60 (m, H-9); 1,60 (d; 2,2 Hz, H-10); 1,18 (s, H-12); 0,99 (s, H-13); 0,97 (s, H-14); 4,85(d; 1,32 Hz, $\mathrm{H}-15) ; 4,96$ (d; $1,32 \mathrm{~Hz}, \mathrm{H}-15) ; \mathrm{RMN}^{13} \mathrm{C}\left(75 \mathrm{MHz}, \mathrm{CDCl}_{3}\right): \delta 50,9$ (C-1); 30,3 (C-2); 39,3 (C-3); 59,6 (C-4); 63,7 (C-5); 29,9 (C-6); 30,0 (C-7); 151,9 (C-8); 48,8 (C-9); 39,9 (C-10); 34,0 (C-11); 17,0 (C-12); 27,3 (C-13); 21,9 (C-14); 112,9 (C-15).

\section{Caracterização do piscidinol A (2) ${ }^{10,11}$}

Cristais incolores, P.F. $198-200{ }^{\circ} \mathrm{C},[\alpha]_{\mathrm{d}}=-62,1^{\circ}\left(\mathrm{CH}_{2} \mathrm{Cl}_{2} ; c\right.$ 0,024); IV (KBr) $v_{\max }\left(\mathrm{cm}^{-1}\right): 3.570,3.374,2.881,2.982,1.697$. EMIE $(\mathrm{m} / \mathrm{z}): \mathrm{m} / \mathrm{z} 476[\mathrm{M}+2]^{+}, 457$ (21), 441 (19), 398 (11), 384 (22), 369 (100), 365 (13), 351 (18), 325 (33), 205 (20) e 59 (35). RMN ${ }^{1} \mathrm{H}\left(300 \mathrm{MHz}, \mathrm{CDCl}_{3}\right): \delta 1,40(\mathrm{~m}, \mathrm{H}-1) ; 1,96(\mathrm{~m}, \mathrm{H}-1) ; 2,20(\mathrm{t} ; 3,3$ $\mathrm{Hz} ; 14,4 \mathrm{~Hz}, \mathrm{H}-2$ ); 2,71 (dd; 5,4 Hz; 14,4 Hz, H-2); 1,73 (m, H-5); 2,06(m, H-6); 5,29 (dd; 3,3 Hz; 10,5 Hz, H-7); 2,25 (m, H-9); 1,51 (m, H-11); 1,62 (m, H-12); 1,82 (m, H-12); 1,45 (m, H-15); 1,47 (m, H-16); 2,00 (m, H-16); 1,48 (m, H-17); 0,81 (s, H-18); 1,00 (s,
H-19); 1,34 (m, H-20); 0,91 (d; 6,0 Hz, H-21); 1,18 (m, H-22); 1,84 (m, H-22); 4,09 (m, H-23); 3,15 (d; 8,1 Hz, H-24); 1,30 (s, H-26); 1,28 (s, H-27); 1,03 (s, H-28), 1,10 (s, H-29); 0,99 (s, H-30); 2,82 (sl, $\mathrm{OH}) ; 2,78(\mathrm{sl}, \mathrm{OH}) ; \mathrm{RMN}{ }^{13} \mathrm{C}\left(75 \mathrm{MHz}, \mathrm{CDCl}_{3}\right): \delta 38,5(\mathrm{C}-1) ; 34,9$ (C-2); 217,0 (C-3); 47,8 (C-4); 52,3 (C-5); 24,3 (C-6); 117,9 (C-7); 145,7 (C-8); 48,4, (C-9); 35,0 (C-10); 18,3 (C-11); 33,8 (C-12); 43,5 (C-13); 51,2 (C-14); 34,0 (C-15); 28,4 (C-16); 53,8 (C-17); 22,0 (C18); 12,8 (C-19); 33,7 (C-20); 18,9 (C-21); 40,5 (C-22); 69,7 (C-23); 75,0 (C-24); 74,3 (C-25); 27,4 (C-26); 26,2 (C-27); 24,5 (C-28); 21,6 (C-29); 27,4 (C-30).

\section{Caracterização da 9-metóxi-cantin-6-ona $(3)^{8,12}$}

Cristais amarelos, P.F. $175-176{ }^{\circ} \mathrm{C}$ (lit $\left.{ }^{12} 175-176{ }^{\circ} \mathrm{C}\right), \mathrm{IV}(\mathrm{KBr})$ $v_{\max }\left(\mathrm{cm}^{-1}\right): 1.667,1.633,1.608,1.493,1.275,1.223,1.152,1.030$, 844, 815, 619, 547. EM-IE (m/z): 250 [M+] (100), 235 (1,3), 221 (21,3), 207 (32,0), $192(4,0), 179(17,3), 153(9,3), 126(6,6), 125$ $(8,0)$. RMN ${ }^{1} \mathrm{H}\left(200 \mathrm{MHz}, \mathrm{CDCl}_{3}\right): \delta 7,83(\mathrm{~d} ; 5,2 \mathrm{~Hz}, \mathrm{H}-1) ; 8,75$ (d; 5,2 Hz, H-2); 8,00 (d; 10,0 Hz, H-4), 6,94 (d; 10,0 Hz, H-5); 8,18 (d; 2,4 Hz, H-8); 7,06 (dd; 2,4 Hz; 8,5 Hz, H-10); 7,93 (d; 8,5 Hz, $\mathrm{H}-11) ; 3,98$ (s, OMe); RMN ${ }^{13} \mathrm{C}\left(50 \mathrm{MHz}, \mathrm{CDCl}_{3}\right): \delta 115,7(\mathrm{C}-1)$; 146,1 (C-2); 140,0 (C-4); 128,7 (C-5); 159,9 (C-6); 101,5 (C-8); 162,7 (C-9); 114,3 (C-10); 123,5 (C-11); 117,3 (C-12); 141,4 (C-13); 130,7 (C-14); 132,5 (C-15); 135,8 (C-16); 56,1 (OMe).

\section{RESULTADOS E DISCUSSÃO}

A separação cromatográfica do extrato hexânico dos galhos de Simaba guianensis subsp. ecaudata resultou no isolamento de 103,0 mg de uma substância incolor identificada como óxido de cariofileno. $\mathrm{O}$ espectro de IV mostrou as absorções em $3.066 \mathrm{~cm}^{-1}$, indicando estiramento C-H de carbonos insaturados, $2.950,2.932$ e $2.861 \mathrm{~cm}^{-1}$ (estiramento $\mathrm{C}-\mathrm{H}$ de carbonos saturados), $1.629 \mathrm{~cm}^{-1}$ referentes ao estiramento $\mathrm{C}=\mathrm{C}$. As análises dos espectros de $\mathrm{RMN}{ }^{1} \mathrm{H}$ e ${ }^{13} \mathrm{C}$ e bidimensionais COSY, HMQC e HMBC estão de acordo com os dados publicados para o óxido de cariofileno. ${ }^{9}$

O fracionamento cromatográfico do extrato hexânico dos galhos de $S$. guianensis subsp. ecaudata resultou no isolamento de 164,7 mg de uma substância cristalina e incolor, com P.F. $198-200{ }^{\circ} \mathrm{C}$ e $[\alpha]_{\mathrm{d}}=-62,1^{\circ}\left(\mathrm{CH}_{2} \mathrm{Cl}_{2} ; c 0,024\right)$, que estão de acordo com os dados publicados para o triterpeno piscidinol A (2). ${ }^{10,11} \mathrm{~A}$ identificação foi confirmada pela análise do espectro de IV que indicou absorções em 3.570 e $3.374 \mathrm{~cm}^{-1}$ de estiramento O-H, 2.881 a $2.982 \mathrm{~cm}^{-1}$ de estiramento $\mathrm{C}$-H de alifáticos e $1.697 \mathrm{~cm}^{-1}$ intenso, sugerindo estiramento de carbonila de cetona. As análises dos espectros de $\mathrm{RMN}{ }^{1} \mathrm{H}$ e ${ }^{13} \mathrm{C}$ e bidimensionais COSY, HMQC e HMBC permitiram confirmar a substância isolada como sendo o piscidinol-A. ${ }^{10,11}$

O fracionamento cromatográfico da fração clorofórmica também resultou no isolamento de um alcaloide identificado como 9-metóxi-cantin-6-ona (3). ${ }^{12} \mathrm{O}$ alcaloide apresentou-se como cristais amarelos com P.F. $175-176{ }^{\circ} \mathrm{C}$ e espectro de massas compatíveis com dados descritos na literatura. ${ }^{8,12} \mathrm{As}$ análises dos espectros de $\mathrm{RMN}{ }^{1} \mathrm{He}$ e ${ }^{13} \mathrm{C}$ e bidimensionais COSY, HMQC e HMBC permitiram confirmar a substância isolada como sendo 9-metóxi-cantin-6-ona.

No fracionamento que levou ao isolamento da 9-metóxi-cantin-6-ona foi isolada outra substância, um sólido de cor amarelo-laranja, solúvel em $\mathrm{MeOH}$, com ponto de fusão bem distinto da 9-metóxi-cantin-6-ona (decompõe a $232{ }^{\circ} \mathrm{C}$ ) e que também testou positivo para alcaloides na análise por CCD com o reagente de Dragendorff.

$\mathrm{O}$ espectro de IV apresentou uma banda intensa em $3.447 \mathrm{~cm}^{-1}$, característica de presença de grupamento hidroxila, frequências de estiramento C-H de carbono insaturado em 3.096 e $3.021 \mathrm{~cm}^{-1}$, estiramento de carbonila $\alpha$-insaturada em $1.660 \mathrm{~cm}^{-1}$, estiramento $\mathrm{C}=\mathrm{C}$ em $1.619 \mathrm{~cm}^{-1}$ e uma absorção intensa em $837 \mathrm{~cm}^{-1}$ de deformação 
angular característica de substâncias aromáticas. O espectro de UV (MeOH) apresentou $\lambda_{\max }(\log$ e) em $206(3,00), 216(1,81), 309$ $(0,40)$ e $355(0,56)$.

No espectro de $\mathrm{RMN}{ }^{1} \mathrm{H}$ foi observado um sinal em $\delta 3,90$ que correlaciona no HMQC com o sinal em $\delta 56,5$, sendo característico de uma metoxila. O hidrogênio em $\delta 7,74$ correlaciona com o carbono em $\delta 102,1$ no HMQC e acopla em relação meta com o hidrogênio em $\delta 6,96$. Este acopla em relação orto com o hidrogênio em $\delta 7,89$ e correlaciona no HMBC com o sinal em $\delta$ 164,3. Esse carbono corresponde ao carbono ligado à metoxila, pois também correlaciona a longa distância com o hidrogênio em $\delta 3,90$. Apesar de não ser observado um sinal típico de hidrogênio de ácido, acima de $\delta 12,0$, o espectro de IV mostra claramente a presença de uma função hidroxila na molécula através da banda em $3.447 \mathrm{~cm}^{-1}$, que sugere se tratar de um alcaloide $\beta$-carbolínico com carbonila $\alpha$-insaturada de ácido.

$\mathrm{O}$ hidrogênio em $\delta 6,88$ acopla com o hidrogênio em $\delta$ 7,93 com constante de acoplamento em $9,5 \mathrm{~Hz}$, confirmado por correlação no espectro de COSY, sugerindo que estes estejam em relação cis. O hidrogênio com $\delta 6,88$ apresenta correlação no HMBC com o carbono em $\delta 160,3$ que corresponde a uma carbonila. Todos os deslocamentos químicos de hidrogênio e carbono são descritos na Tabela 1, juntamente com as correlações correspondentes observadas a longa distância no espectro de HMBC.

Tabela 1. Deslocamentos químicos de RMN ${ }^{1} \mathrm{H}(500 \mathrm{MHz}), \mathrm{RMN}{ }^{13} \mathrm{C}(125$ $\mathrm{MHz}$ ) e correlações C-H J $\mathrm{J}^{1}$ (HMQC) e J $\mathrm{J}^{3-4}$ (HMBC) para a substância (4) (em MeOD-d4)

\begin{tabular}{cccc}
\hline Posição & $\delta^{13} \mathrm{C}$ & $\delta^{1} \mathrm{H}(\mathrm{m}, \mathrm{J}(\mathrm{Hz}))$ & $\mathrm{HMBC}\left(\mathrm{J}^{2,3}\right)$ \\
\hline 4 & 117,4 & $7,94(\mathrm{~d}, 5,0)$ & 145,$3 ; 133,0$ \\
3 & 145,3 & $8,62(\mathrm{sl})$ & - \\
$3^{\prime}$ & 134,4 & $7,93(\mathrm{~d}, 9,5)$ & - \\
2 & 130,3 & $6,88(\mathrm{~d}, 9,5)$ & 160,$3 ; 134,4$ \\
1 & 160,3 & - & - \\
8 & 125,4 & $7,89(\mathrm{~d}, 8,5)$ & 142,$4 ; 117,8$ \\
7 & 115,1 & $6,96(\mathrm{dd}, 8,5,2,0)$ & 164,$3 ; 102,1$ \\
6 & 164,3 & - & - \\
5 & 102,1 & $7,74(\mathrm{~d}, 2,0)$ & 164,$3 ; 142,4 ; 115,1 ;$ \\
& & & 117,8 \\
$4 \mathrm{~b}$ & 117,8 & - & - \\
$8 \mathrm{a}$ & 142,4 & - & - \\
$4 \mathrm{a}$ & 133,0 & - & - \\
$9 \mathrm{a}$ & 132,5 & - & 164,3 \\
1 & 134,4 & - & - \\
OMe & 56,5 & $3,90(\mathrm{~s})$ & \\
\hline
\end{tabular}

Os deslocamentos químicos observados sugerem a posição da metoxila em C-6, através das constantes de acoplamento entre os hidrogênios H-7 $(2,0 \mathrm{~Hz})$ e H-5 $(2,0 \mathrm{~Hz})$ e ainda o acoplamento com relação orto do hidrogênio H-7 $(8,5 \mathrm{~Hz})$ com H-8 $(8,5 \mathrm{~Hz})$. As correlações heteronucleares $\mathrm{C}-\mathrm{H}$ a longa distância entre o hidrogênio H-7 e C-6, H-5 e C-6 sugerem tratar-se do ácido 6-metóxi-(9H- $\beta$ carbolin-1-il)-(Z)-2-propenoico, isolado pela primeira vez como um produto natural.

O espectro de massas da substância obtido inicialmente por CGEM com impacto eletrônico a 70 eV não apresentou o íon molecular, apenas as fragmentações com $\mathrm{m} / \mathrm{z}$ de 250 (100), 235 (7), 221 (30), 207 (54), 192 (19), 179 (57) e 153 (27). A ausência do íon molecular no espectro de massas pode ser explicada pela facilidade de perda de uma molécula de água durante a fragmentação da molécula, gerando o pico base de $\mathrm{m} / \mathrm{z}, 250$. Posteriormente, uma análise por CL-EM empregando um espectrômetro de massas com ionizador do tipo triplo quadrupolo com ionização tipo APCI permitiu a visualização do íon quase molecular com $\mathrm{m} / \mathrm{z}$ 267,20, acompanhado do íon fragmentário $\mathrm{m} / \mathrm{z}$ 250,52 , resultante da perda de $\mathrm{H}_{2} \mathrm{O}$ (Figura 2). Esses dados confirmam a substância como sendo o ácido 6-metóxi-(9H- $\beta$-carbolin-1-il)-(Z)2-propenoico. Esse ácido é um precursor hipotético de alcaloides do tipo cantinona. De acordo com os nossos levantamentos, é o primeiro relato da ocorrência natural dessa substância.

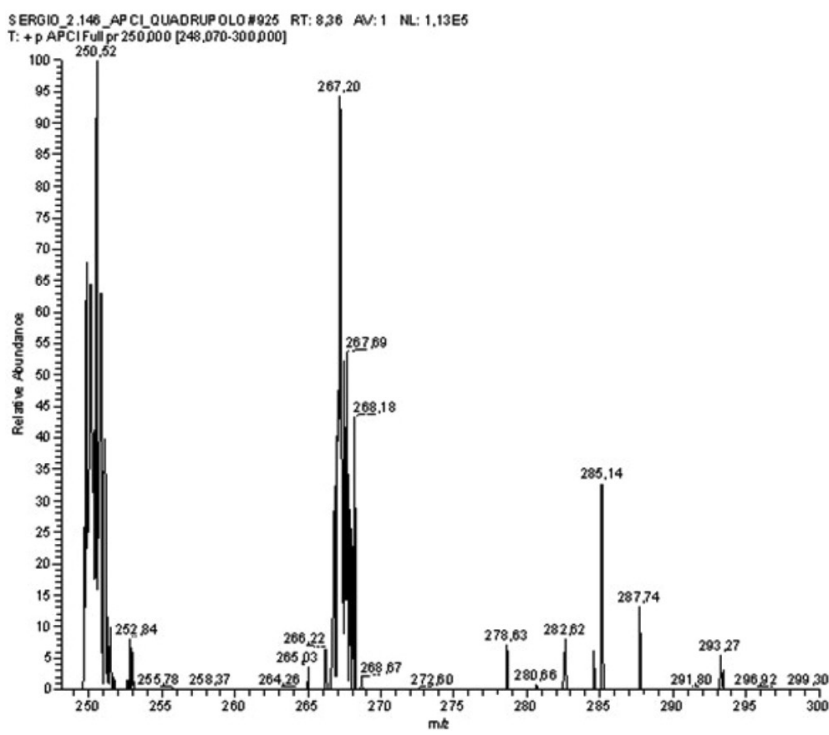

Figura 2. Espectro de massas do ácido 6-metóxi-(9H- $\beta$-carbolin-1-il)-(Z)-2-propenoico, obtido em triplo quadrupolo com fonte de ionização tipo APCI

\section{Quantificação de 9-metóxi-cantin-6-ona em extratos e frações dos galhos de $S$. guianensis subesp. ecaudata}

A análise quantitativa por CLAE de constituintes químicos isolados de plantas é uma ferramenta de grande auxílio, não somente para a verificação dos componentes de uma planta como para a validação da planta quando esta é utilizada tradicionalmente na medicina popular.

Os alcaloides do tipo cantinona são importantes marcadores quimiotaxonômicos das espécies de Simaroubaceae, em especial para espécies do gênero Simaba. Previamente, o grupo do Prof. Otto R. Gottlieb iniciou uma série de publicações em The Chemistry of Brazilian Simaroubaceae, relatando os constituintes químicos de espécies de Simaroubaceae, com a descrição de 8-metóxi-cantin-6-ona e 3-metóxi-cantin-2,6-diona. ${ }^{11}$ Posteriormente, Saraiva e colaboradores ${ }^{8}$ descreveram a presença de 9-metóxi-cantin-6-ona de S. polyphylla. Mais recentemente, Almeida e colaboradores ${ }^{13}$ descreveram a presença de 4-metóxi-cantin-6-ona de S. ferruginea.

A partir do isolamento de 9-metóxi-cantin-6-ona foi realizada a sua quantificação em diferentes extratos de $S$. guianensis, devido à sua importância quimiotaxonômica e sua significativa atividade citotóxica, determinada em ensaios in vitro contra células tumorais tipo MCF-7 (câncer de mama) e A-549 (câncer de pulmão), nas concentrações de 4,5 e inferior a $2,5 \mu \mathrm{g} / \mathrm{mL}$, respectivamente. ${ }^{14}$

O padrão da substância 9-metóxi-cantin-6-ona foi purificado diretamente da fração clorofórmica dos galhos de S. guianensis subesp. ecaudata, empregando-se técnicas cromatográficas e de recristalização. A Figura 3 apresenta a identificação do padrão isolado no extrato aquoso de $S$. guianensis pelo método de adição de padrão.

$\mathrm{Na}$ análise por CLAE dos extratos metanólicos, foi necessário realizar um tratamento por extração em fase sólida (EFS), conforme 


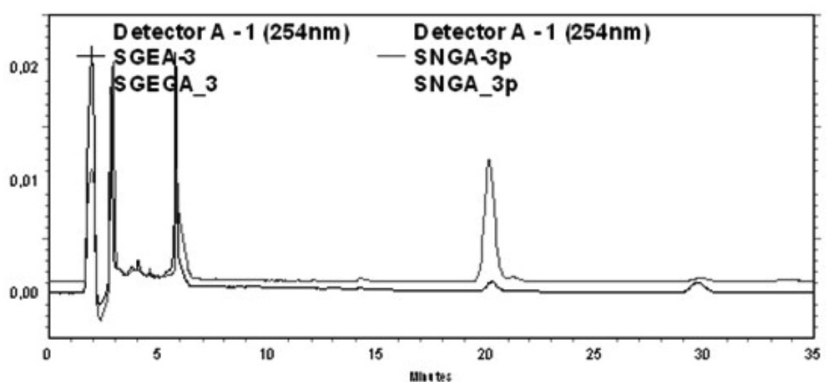

Figura 3. Cromatogramas da fração 3 do extrato aquoso de Simaba guianensis subsp. ecaudata com (cinza) e sem coinjeção (preto) de padrão 9-metóxi-cantin-6-ona

descrito anteriormente. As três frações obtidas através do tratamento por EFS foram analisadas por CLAE e foi possível detectar a 9-metóxi-cantin-6-ona apenas na fração eluída com mistura $\mathrm{MeOH} /$ AcOEt (7:3) (Figura 4).

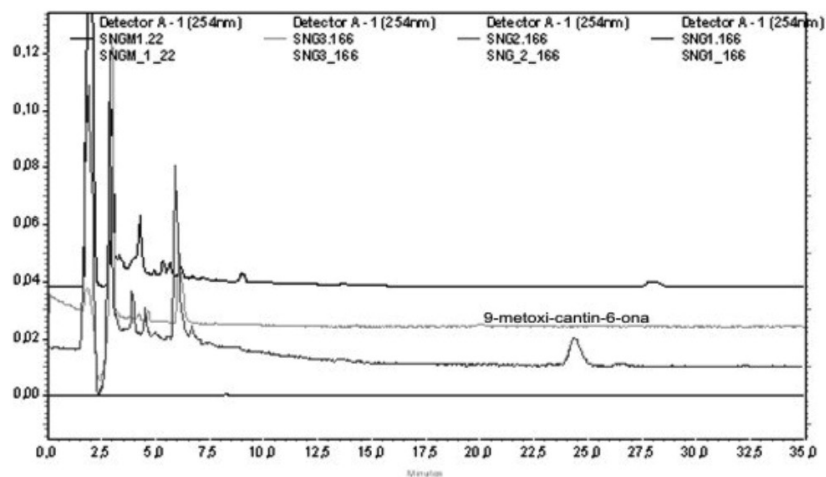

Figura 4. Cromatogramas dos extratos metanólicos dos galhos de Simaba guianensis subesp. ecaudata; SNGM_1_22, corresponde ao extrato metanólico sem tratamento por EFS, as demais após tratamento por EFS:

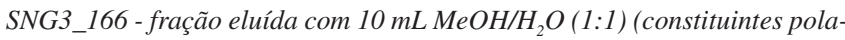
res); SNG_2_166 - fração eluída $2 \mathrm{~mL}$ de MeOH/EtOAc (7:3) (fração com 9-metóxi-cantin-6-ona); SNG1_166 - fração eluída com $10 \mathrm{~mL}$ de $\mathrm{MeOH} /$ EtOAc (7:3) (fração apolar)

Amostras do extrato aquoso dos galhos $(5,0 \mathrm{mg} / \mathrm{mL})$ de $S$. guianensis também foram submetidas ao tratamento por EFS, antes de ser analisado por CLAE, que resultaram em quatro frações analisadas. As análises das frações eluídas com 1,0 mL de MeOH/AcOEt (7:3) confirmaram a presença de 9-metóxi-cantin-6-ona na fração 3 e essa fração foi utilizadas na análise quantitativa do alcaloide no extrato aquoso (Figura 5).

A fração clorofórmica dos galhos de $S$. guianensis foi analisada diretamente por CLAE, sem necessidade de pré-tratamento por EFS.

A curva de calibração obtida para a quantificação de 9-metóxi-cantin-6-ona apresentou excelente linearidade $\left(\mathrm{R}^{2}=0,9997\right)$, nas faixas de concentrações analisadas $(n=5)$. A precisão da análise (expressa em CV \%) foi bastante satisfatória (Tabela 2), mesmo para as amostras submetidas a tratamento por EFS. Todas as amostras foram analisadas em duplicata, calculando-se em seguida o valor médio das áreas obtidas de cada amostra.

Em seguida, a 9-metóxi-cantin-6-ona presente nos diferentes extratos e frações analisados foi quantificada e os valores expressos em percentuais de peso (peso cantinona/peso seco de extrato ou fração).

As concentrações de 9-metóxi-cantin-6-ona obtidas foram baixas, especialmente no extrato aquoso. O extrato metanólico e fração clorofórmica apresentaram quantidades maiores de 9-metóxi-cantin-6-ona. Esses resultados já eram esperados devido à menor polaridade

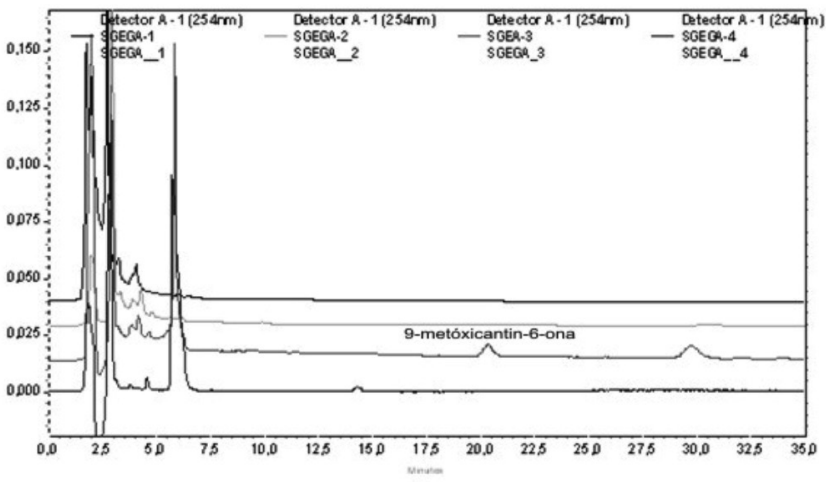

Figura 5. Cromatogramas das frações de EFS obtidas do extrato aquoso dos galhos de S. guianensis subsp. ecaudata. SGEGA_1 - fração 1 eluída com $10 \mathrm{~mL}$ de $\mathrm{H}_{2} \mathrm{O}$; SGEGA-2 - fração 2 eluída com $10 \mathrm{~mL}$ de $\mathrm{MeOH} / \mathrm{H}_{2} \mathrm{O}$ (1:1); SGEGA_3 -fração 3 eluída com $1 \mathrm{~mL}$ do sistema MeOH/AcOEt (7:3) (fração com 9-metóxi-cantin-6-ona); SGEGA-4 - fração 4 eluída com $10 \mathrm{~mL}$ de lavagem com $\mathrm{MeOH} / \mathrm{AcOEt}$ (7:3)

Tabela 2. Resultado da quantificação por EFS-CLAE de 9-metóxi-cantinona presente nos extratos e frações dos galhos de $S$. guianensis subesp. ecaudata

\begin{tabular}{lccc}
\hline Amostra & $\mathrm{CV}(\%)$ & $\mathrm{C}(\mu \mathrm{g} / \mathrm{mL})$ & $\begin{array}{c}\text { Relação } \\
\mathrm{p} / \mathrm{p}(\%)\end{array}$ \\
\hline Extrato aquoso de $S$. guianensis & 3,95 & 0,2664 & 0,00053 \\
Extrato metanólico de $S$. guianensis & 3,53 & 0,7096 & 0,14 \\
Fração clorofórmica de S. guianensis & 6,18 & 0,7757 & 0,62 \\
\hline
\end{tabular}

de 9-metóxi-cantin-6-ona, que é extraída com maior facilidade com solventes orgânicos, tais como metanol e clorofórmio. Como citado anteriormente, a concentração em que 9-metóxi-cantin-6-ona apresenta atividade citotóxica é de 2,0 a $4,5 \mu \mathrm{g} / \mathrm{mL} .{ }^{12}$ Para que o extrato aquoso apresentasse atividade citotóxica seria necessário que esse alcaloide estivesse presente em concentrações em torno de 100 a 200 vezes maiores.

\section{CONCLUSÃO}

As substâncias isoladas dos galhos de $S$. guianensis subesp. ecaudata são conhecidas por apresentarem citotoxicidade descritas na literatura. $\mathrm{O}$ triterpeno piscidinol $\mathrm{A}$ apresenta atividade contra células P388 e KB com $\mathrm{CI}_{50}=1,2$ e $5,0 \mu \mathrm{g} / \mathrm{mL}$, respectivamente, e o alcaloide 9-metóxi-cantin-6-ona mostrou-se citotóxico para células de câncer de mama e de pulmão. ${ }^{14,15}$

$\mathrm{O}$ isolamento de um triterpeno do tipo tirucalano com insaturação na posição C-7, como registrado nesse trabalho, é comum em espécies da família Simaroubaceae, visto que triterpenos dessa classe são precursores de quassinoides, que são os principais marcadores quimiotaxonômicos dessa família. ${ }^{16}$ A presença de alcaloides do tipo cantinona e beta-carbolínicos também são comuns em espécies da família, ${ }^{17}$ contudo essa é a primeira vez que o alcaloide 4 é descrito.

A quantificação de 9-metóxi-cantin-6-ona nos extratos e frações dos galhos de $S$. guianensis subesp. ecaudata demonstra que a concentração desse alcaloide citotóxico não é suficiente para conferir toxidez ao extrato aquoso dessa espécie. Contudo, isto só pode ser confirmado através de ensaios de toxicidade aguda e crônica in vivo.

\section{AGRADECIMENTOS}

Ao CNPq, pela concessão de bolsas e auxílio à pesquisa pelos programas PNOPG/CNPq (Proc. N. 55026/01-3) e CT-Amazonia (Proc. N. 
575503/2008-4 e Proc. N. 575625/2008-2). À M. Souza pela análise por CL-EM do ácido 6-metóxi- (9H- $\beta$-carbolin-1-il)-(Z)- prop-2-enoico.

\section{REFERÊNCIAS}

1. Ribeiro, J. E. L. S.; Hopkins, M. J. G.; Vicentini, A.; Sothers, C. A.; Costa, M. A. S.; Brito, J. M.; Souza, M. A. D.; Martins, L. H. P.; Lohmann, L. G.; Assunção, P. A. C. L.; Pereira, E. C.; Silva, C. F.; Mesquita, M. R.; Procópio, L. C.; Flora da Reserva Ducke - Guia de identificação das plantas vasculares de uma floresta de terra-firme na Amazônia Central, INPA: Manaus, 1999, p. 547-549.

2. Vieira, I. J. C.; Braz-Filho, R.; Rodrigues Filho, E.; Vieira, P. C.; Silva, M. F. G. F.; Fernandes, J. B.; J. Braz. Chem. Soc. 1999, 10, 76.

3. Ozeki, A.; Hitotsuyanagi, Y.; Hashimoto, E.; Itokawa, H.; Takeya, K.; Alves, S. M.; J. Nat. Prod. 1998, 61, 776.

4. Muhammad, H.; Bedir, E.; Khan, S. I.; Tekwani, B. L.; Khan, I. A.; Takamatsu, S.; Pelletier, J.; Walker, L. A.; J. Nat. Prod. 2004, 67, 772.

5. Barbosa, L. F.; Braz-Filho, R.; Vieira, I. J. C.; Chem. Biodivers. 2011, 8, 2163.

6. Da Silva, M. F. G. F.; Gottlieb, O. R.; Biochem. Syst. Ecol. 1987, 15, 805.

7. Cavalcante, P. B.; Revisão taxonômica do gênero Simaba Aubl. (Simaroubaceae) na América do Sul, Museu Paraense Emílio Goeldi: Belém, 1983, p. 85.
8. Saraiva, R. C. G.; Pinto, A. C.; Nunomura, S. M.; Pohlit, A. M.; Quim. Nova 2006, 29, 264.

9. Krebs, K. G.; Ruber, H. E.; Arzneim. Forsch. 1960, 10, 500.

10. Gray, A. I.; Bhandari, P.; Waterman, P. G.; Phytochemistry 1988, 27, 1808.

11. Tinto, W. F.; Jagessar, P. K.; Ketwaru, P.; Reynolds, W. F.; McLean, S.; J. Nat. Prod. 1991, 54, 972.

12. Giesbrecht, A. M.; Gottlieb, H. E.; Gottlieb, O. R.; Goulart, M. O. F.; de Lima, R. A.; Sant'ana, A. E. G.; Phytochemistry 1980, 19, 313.

13. Almeida, E. S. S.; Cechinel-Filho, V.; Niero, R.; Clasen, B. K.; Balogun, S. O.; Martins, D. T. O.; J. Ethnopharmacol. 2011, 134, 630.

14. Kuo, P.; Shi, L.; Amooru, G. D.; Su, C.; Huang, C. Q. C.; Wu, J.; Lin, A.; Bastow, K. F.; Lee, K.; Wu, T.; J. Nat. Prod. 2003, 66, 1324

15. Itokawa, H.; Kishi, E.; Morita H.; Takeya, K.; Chem. Pharm. Bull. 1992, 40, 1053.

16. Dewick, P. M.; Medicinal Natural Products (A Biosynthetic Approach), John Wiley \& Sons Ltd Ed.: Baffins Lane, Chichester, West Sussex, 1997, p. 195, 199, 207.

17. Ohmoto, T.; Koike, K.; The Alkaloids, Arnold Brom Ed: New York, 1989, p. 135 